follows. Matrix methods are applied to the kinematics of a number of complicated spatial mechanisms in analyses which are presented in detail: in addition to their purely illustrative function these examples contain original results by the author which should be of permanent value.

A short chapter on design is followed by a section on the dynamics of mechanisms, including inertia forces and balancing. The topics, although given a rather uneven general treatment, are well supported by original worked examples. Finally, matrix methods are applied to problems of machining complicated surfaces, chiefly gears, and to the optics of mirrors and scanners.

The text is illustrated generously. There are no set questions for use by students and the book is definitely not for beginners. But specialists will find much of value in it.

L. MAUNDer

\section{AUTOMATIC CONTROL}

Fundamentals of Automatic and Remote Control By S. A. Ginzburg, I. Ya. Lekhtman and V. S. Malov. Translated by R. Farkas and J. J. Schorr-Kon. English translation edited by J. M. Howl. (International Series of Monographs in Automation and Automatic Control, Vol. 7.) Pp. xiv +498 . (London and New York: Pergamon Press, Ltd., 1966.) 100s. net.

WrTH the "knowledge explosion" in control engineering, it is no more possible to write a moderately sized text-book to encompass the entire field than to write a satisfactory introduction to European history. The authors of Fundamentals of Automatic and Remote Control write for an audience which was not trained in the field and attempt to explain how and what the various components in a control system do; what they deal with they explain well. They stress components and devices such as transducers, tachometers and selsyns as well as some basic theory such as polar plots and step response.

Unfortunately, there is much left out. The authors state in their preface that "description is confined to electrical components and systems; mechanical, hydraulic and pneumatic systems employed in automation have been oraitted through limitations of space". Fair enough, but the omissions include references to control theory after the mid-fifties, such as the maximum principle and dynamic programming; cybernetics is given a one line mention and this only in connexion with computers and not with control systems as such; Lyapunov receives one line without a hint as to the connexion of his methods with those discussed in the book and there is no mention of controls with regard to non-deterministic inputs. Moreover, what is discussed is confined almost entirely to linear systems, so that the reader may be given a false impression of the generality of the principles enunciated. In short, there is no hint, let alone a discussion, about the powerful abstract basis of control theory. Because no dates are given for the original Russian text, it must be assumed that this book is at least ten years old.

Paul Alper

\section{HYDRAULIC TURBINES}

\section{Hydroturbines}

Design and Construction. By N. N. Kovalev. Translated from the Russian by M. Segal. Pp. vi +682. (Jerusalem: Israel Program for Scientific Translations; London: Oldbourne Press, 1965.) 153s.

Or all the prime movers, water turbines have been the most neglected in English technical literature. There are no standard works of reference dealing with mechanical design and little is being published. In the Soviet Union, with its vast resources of water power, a national water turbine industry has been established, and several volumes rather similar in scope and presentation to that by Kovalev have appeared in the last decade. Parts of these have been translated in Britain under the auspices of the Department of Scientific and Industrial Research as it used to be, so that some of the contents of this volume are already familiar. Nevertheless, this is the first exhaustive design manual for water turbines to have become generally available in English.

The manual is chiefly concerned with the vertical shaft Kaplan and Francis turbines. The coverage is impressive. As well as the discussion of construction and detailed calculation of components of these machines, the relative costs of many design variants are compared and the variants themselves are shown in considerable detail. Much of the value of the book lies in its hundreds of design sketches, both of major components and of such details as sealing arrangements. These convey the design concopt better than any amount of descriptive matter, and are taken from a wide range of manufactured turbines.

Although the basis on which the relative costs of components are considered is sound, there are occasions, which can only be accounted for by scarcity, when a premium seems to be put on the use of machine tools with an accompanying disregard of the costs of manual labour. Some of the cost analyses used in justifying the choice of an engineering solution are, therefore, not applicable in British conditions. Most of the design problems dealt with are fairly conventional, but there are several exceptions such as the comments on the construction of fabricated (welded) shafts, the notes on methods of manufacturing split Francis runners, and the chapter on the use of water lubricated rubber lined bearings. The methods used in calculating components follow a familiar pattern in that an approximate solution is usually looked for, and the analysis is often verified by plant tests. Although most of these methods are well tried, some are novel and must therefore be regarded with caution. The methods suggested for calculating stresses in runner blades of medium head Francis runners are in this category, for it is difficult to imagine that a runner blade-a shape of considerable complexity - can be satisfactorily approximated to by a simple curved bar. On the other hand, this simplification does give a workable method of calculating stress which can be confirmed by plant tests and which therefore provides a solution where very little was available before. On the other hand, it is good to see a relatively simple method for dealing with the high localized stresses at the junction of spiral casing and speed ring; normally one needs a computer for predicting them, and it may be that the formulae given will make it possible to do this more simply.

Although much of the book is taken up by methods of calculation of Kaplan runners, there are no details of analytical or even numerical methods for determining the stresses in Kaplan runner blades anywhere else but at the blade flange. On the other hand, there are detailed site measurements of stresses under various conditions in a very large Kaplan vane. Similarly, no attempt is made to show how to determine the bending stresses and deformations in a speed ring. Where the complexity of the problem is such that a mathematical solution is not readily available, Kovalev prefers to rely on an empirical rather than an analytical approach.

The chapters on the hydraulic aspects of water turbines are more than adequate, but cover much the same ground as many available handbooks. One interesting addition is an account of how water turbines have been standardized in the Soviet Union. The various model runners available are listed, together with graphs showing what they can do when made in standard sizes and set to work under a range of suitable heads. Standardization is one of the important problems of the industry, and could be solved only by a single authority prescribing the solution 